\title{
BMJ Open Barriers and facilitators to recruitment of South Asians to health research: a scoping review
}

\author{
Teo AW Quay, ${ }^{1,2}$ Leora Frimer, ${ }^{3,4}$ Patricia A Janssen, ${ }^{4,5,6}$ Yvonne Lamers ${ }^{2,5,6}$
}

To cite: Quay TAW, Frimer L, Janssen PA, et al. Barriers and facilitators to recruitment of South Asians to health research: a scoping review. BMJ Open 2017;7:e014889. doi:10.1136/ bmjopen-2016-014889

- Prepublication history and additional material for this paper are available online. To view these files please visit the journal online (http://dx.doi.org/ 10.1136/ bmjopen-2012-014889).

Received 26 October 2016 Revised 17 March 2017 Accepted 31 March 2017

\section{(a) CrossMark}

${ }^{1}$ The Canadian Agency for Drugs and Technologies in Health, Ottawa, ON, Canada

${ }^{2}$ Food Nutrition and Health Program, Faculty of Land and Food Systems, The University of British Columbia, Vancouver, BC, Canada

${ }^{3}$ Departments of Epidemiology, Biostatistics and Occupational Health, Faculty of Medicine,

McGill University, Montreal, QC, Canada

${ }^{4}$ School of Population and Public Health, Faculty of Medicine, The University of British Columbia, Vancouver, BC, Canada ${ }^{5}$ British Columbia Children's Hospital Research Institute, Vancouver, BC, Canada

${ }^{6}$ Women's Health Research Institute, Vancouver, BC, Canada

Correspondence to

Dr. Yvonne Lamers; yvonne.

lamers@ubc.ca

\section{ABSTRACT}

Objectives People of South Asian ethnicity are underrepresented in health research studies. The objectives of this scoping review were to examine the barriers and facilitators to recruitment of South Asians to health research studies and to describe strategies for improving recruitment.

Design Scoping review

Methods Using the Arksey and 0'Malley framework for scoping reviews, we comprehensively searched electronic databases (MEDLINE via PubMed, Cochrane Library, CINAHL and PsycINFO). Studies that identified barriers and facilitators to recruitment, or recruitment strategies for South Asian populations were included. Recruitment barriers, facilitators and strategies were grouped thematically and summarised narratively.

Synthesis of 1846 potentially relevant articles, 15 met the inclusion criteria and were included in the thematic synthesis. Multiple facilitators and barriers to enrolment of South Asians in health research studies were identified; these most commonly related to logistical challenges, language and cultural barriers, concerns about adverse consequences of participating and mistrust of research. Several actionable strategies were discussed, the most common being engagement of South Asian communities, demonstration of cultural competency, provision of incentives and benefits, language sensitivity through the use of translators and translated materials and the development of trust and personal relationships.

Conclusion There is a growing awareness of the barriers and facilitators to recruitment of South Asian participants to health research studies. Knowledge of effective recruitment strategies and implementation during the grant funding stages may reduce the risk of poor recruitment and representation of South Asians.

\section{INTRODUCTION}

South Asians are the largest ethnic minority in Canada and the UK. ${ }^{12}$ South Asian populations have demonstrated historical and projected growth in these regions. The total South Asian population in Canada is currently estimated at 1.6 million individuals; a quarter of the visible minority population (ie, 'persons who are non-Caucasian in race or non-white in colour and who do not report being Aboriginal' $)^{3}$ and $4.8 \%$ of the total Canadian population. It is projected to
Strengths and limitations of this study

- First scoping review to summarise evidence regarding factors that influence the involvement of South Asian participants in health research

- Comprehensive overview of the volume and characteristics of research published on this topic

- Actionable recruitment strategies and topics for further investigation clearly identified

- Published evidence only available from a few countries and primarily in the clinical trial setting with small sample sizes and variable patient populations, so transferability to other contexts may be limited.

reach 3.2-4.1 million individuals by $2031 .^{4}$ Two-thirds of South Asians in Canada identify as East Indian with smaller proportions identifying as Pakistani, Sri Lankan and Punjabi. ${ }^{1}$ In the UK, the South Asian ethnic group had some of the largest population increases over the 2001-2011 time period (ie, 0.4 million each in the Pakistani and Indian subgroups). ${ }^{2}$

South Asian populations face specific health challenges. An analysis of Canadian Community Health Survey data (2001-2013) concluded that South Asians are more likely to report poor self-rated health than Whites. ${ }^{5}$ A higher proportion of South Asians suffer from health conditions, including type 2 diabetes, cardiovascular disease and asthma than people of European ethnicity, ${ }^{6-10}$ and the rates of non-communicable disease are expected to rise. ${ }^{11}$ South Asians are reported to develop non-communicable diseases at younger ages, despite fewer risk factors (eg, at lower body mass index), than other ethnic groups. ${ }^{61213}$ Mental health is also an emerging area of concern, particularly in immigrant women, in whom a higher risk of postpartum depression has been reported. ${ }^{14} 15$

In the context of healthcare access, low socioeconomic status, ${ }^{16}$ racial and cultural discrimination, ${ }^{17} 18$ geography (eg, distance from healthcare centre, lack of access to 
transportation), ${ }^{19}$ language barriers ${ }^{20}$ and traditional hierarchies within families ${ }^{21}$ have been reported to obstruct optimal healthcare of South Asians, and in particular South Asian women. ${ }^{21}$ Frequently, South Asians maintain traditional religious, dietary and healthcare practices, which may not align with modern Western or allopathic medicine $^{22}$ and clinical research approaches. ${ }^{23}{ }^{24}$ Lack of support from families and communities in seeking healthcare and making healthcare decisions may discourage South Asians from engaging in risk-reducing health behaviours, including participation in research. ${ }^{25}$ Lack of English language proficiency, unfamiliarity with local services and lack of attention to cultural factors by healthcare providers may pose a particular challenge to healthcare service access for female South Asians. ${ }^{15}$

Adequate ethnic minority representation in health research is important to support generalisability of research findings and to enable tailored healthcare for ethnic minorities. ${ }^{26} 27$ However, South Asians are under-represented in research, resulting in healthcare practice based on research with limited external validity for the South Asian context. ${ }^{28}{ }^{29}$ In general, there is a lack of representative population health research comparing minority groups in Canada with European counterparts. ${ }^{28}$ This under-representation also occurs elsewhere; a UK-based analysis reported that while South Asians make up $4.5 \%$ of the total population, they represented on average only $0.6 \%$ of participants in six multicentre randomised controlled trials. ${ }^{230}$ Another review noted that trials assessing cardiovascular outcomes in patients with type 2 diabetes had under-representation of the South Asian population compared with the population proportion in the UK, but over-representation in the USA. $^{31}$ Even studies with sufficient numbers of South Asians may not be truly representative of the heterogeneous subgroups that comprise the broader South Asian population. Diets, lifestyles and baseline health risk are noted to vary across South Asian subgroups based on differences in origin, culture and religion. ${ }^{32}$

To better understand the current knowledge and perspectives on this topic, a scoping review of the evidence regarding barriers and facilitators to recruitment and strategies that have been employed or evaluated to improve representation of South Asians was undertaken. A preliminary search of the literature did not yield sufficient reports on the Canadian population, thus the review was expanded to include literature regarding South Asian populations residing in other countries.

\section{METHODS}

A scoping review was undertaken according to the methods outlined by Arksey and O'Malley and Levac et $a l^{33}{ }^{34}$ The Arksey and O'Malley approach involves the identification of a research question; a search for relevant studies; selection of studies; charting the data; collating, summarising and reporting the results and an optional consultation with stakeholders to inform or validate the
Table 1 Scoping review search terms

\begin{tabular}{|c|c|}
\hline $\begin{array}{l}\text { A. Ethnicity-related search } \\
\text { terms }\end{array}$ & $\begin{array}{c}\text { B. Strategy-related search } \\
\text { terms }\end{array}$ \\
\hline South Asian or South Asia & $\begin{array}{l}\text { Patient selection ([MeSH } \\
\text { term]) }\end{array}$ \\
\hline Minority groups ([MeSH term]) & Recruitment \\
\hline Minority health ([MeSH term]) & Enrolment \\
\hline Sri Lankan or Sri Lanka & Recruitment strategies \\
\hline Bangladeshi or Bangladesh & OR any of the above \\
\hline \multicolumn{2}{|l|}{ Pakistani or Pakistan } \\
\hline \multicolumn{2}{|l|}{ Nepalese or Nepal } \\
\hline \multicolumn{2}{|l|}{ Bhutanese or Bhutan } \\
\hline \multicolumn{2}{|l|}{ Maldivian or Maldives } \\
\hline \multicolumn{2}{|l|}{ Indian or India } \\
\hline \multicolumn{2}{|l|}{ OR any of the above } \\
\hline A an & \\
\hline
\end{tabular}

$\mathrm{MeSH}$, medical subject heading.

findings. ${ }^{33}$ We followed this approach with the exception of the consultation stage, due to limited resources, and employed several suggestions made by Levac et al, including a focused research question, the conduct of thematic synthesis, duplicate study selection and standardised and duplicate extraction. ${ }^{34}$ This methodology was justified given that there is limited knowledge on this topic, and the interest in assessing the depth and breadth of the evidence base. The aim was to identify barriers and facilitators to recruitment of South Asians to health research studies and associated strategies to improve participation. The following specific research questions were addressed:

1. What are the barriers and facilitators to recruitment of South Asian individuals to health research studies?

2. What are the evidence-based strategies for recruitment of South Asian individuals to health research studies?

\section{DATA SOURCES AND SEARCH}

The search strategy was developed and executed by one reviewer (TAWQ). A comprehensive search was conducted using PubMed, the Cochrane Library, CINAHL and PsycINFO. Medical subject headings and keywords including South Asian, minority groups, patient selection, recruitment, enrolment, recruitment strategies and specific South Asian ethnicities were searched from January 2004 through October 2014, and updated in April 2016 (table 1). Search terms are presented in table 1 . The full search string is presented in online supplementary appendix 1.

The search dates were restricted in the interest of identifying the most up-to-date evidence on the topic. The search was supplemented by scanning reference lists of included studies, searching https://clinicaltrials.gov/ and PROSPERO for ongoing work and by a focused 
internet search. Study selection was limited to English language articles or articles that could be translated using Google Translate. Due to the noted limitations of this approach,$^{35}$ translations were to be verified by research staff familiar with the language of publication if possible. Study staff were proficient in German, French, Spanish, Mandarin, Cantonese and several South Asian languages. No restriction was made by publication type. The results from all searches were pooled and deduplicated prior to screening.

\section{Study selection}

All types of studies including primary randomised and non-randomised quantitative studies, qualitative studies, and systematic reviews were included. Commentaries and narrative reviews were excluded to avoid identifying themes from single or few perspectives. We included studies involving South Asian individuals (eg, Sri Lankan, Bangladeshi, Pakistani, Nepalese, Bhutanese, Maldivian and Indian) in any setting or studies involving multiple ethnic groups where South Asians were a specified subgroup or comprised the majority of participants. Studies assessing or reporting on barriers and facilitators to recruitment and recruitment strategies were included. This includes studies assessing the impact or effectiveness, or assessing the comparative impact or effectiveness of barriers, facilitators and recruitment strategies.

One reviewer (TAWQ) independently screened titles and abstracts against the prespecified eligibility criteria. A second reviewer (LF) then screened selected abstracts. Full-text articles were obtained and reviewed by both authors for studies that appeared to meet the eligibility criteria or where eligibility could not be adequately judged. Disagreement was resolved by discussion among the two reviewers with a third reviewer consulted if deemed necessary. Study authors were not contacted for further information.

\section{Data abstraction}

Data abstraction was conducted in duplicate by two authors (TAWQ and LF) using a structured extraction form piloted on two studies. Disagreement was resolved via discussion. Data including (a) study classifiers (lead author, publication year, country), (b) study characteristics (sample size, study design, subject characteristics) and (c) recruitment barriers, facilitators for participation and recruitment strategy data were extracted. Specific descriptions of recruitment barriers, facilitators and strategies were recorded.

Barriers and facilitators, and recruitment strategies were grouped thematically into key topics that emerged on review of the literature. Once established, these themes were presented and discussed narratively. No formal data synthesis or assessment of intervention effectiveness was undertaken. Quality appraisal of selected studies was not conducted as this is not typical of scoping reviews, ${ }^{33}$ but general limitations are discussed. In addition, no formal consultation exercise with stakeholders was conducted.

\section{RESULTS}

\section{Literature search}

The original search on PubMed and Cochrane (2004October 2014) identified 1027 potentially relevant publications. A further 10 studies were identified from grey literature sources. The original search was updated in April 2016 and 199 more potentially relevant articles were identified. Also, the databases CINAHL and PsycINFO were added and these searches (2004-April 2016) identified 645 more potentially relevant articles. After removal of duplicates, a total of 1846 records were screened. All of the publications were available in English, so no translation was required. Based on title and abstract, 1648 records were excluded and 198 full-texts were screened (figure 1). Of the 198 studies included for full-text review, 183 were excluded for various reasons (ie, irrelevant outcomes, study of alternate ethnic groups, review or commentary) and 15 articles met the inclusion criteria and were included in this report ( $\mathrm{n}=9$ from original PubMed search; $n=4$ from PubMed search update; $\mathrm{n}=2$ from CINAHL and PsycINFO).

\section{Study characteristics}

The final 15 articles included in this review focused on South Asian populations and discussed barriers and facilitators for participation in research and potential strategies for recruitment. Primary study characteristics including research area, study populations, sample size and study design are noted in table 2.

Study populations ranged in size from $n=2$ to $n=1319$. The majority of studies were conducted in specific clinical populations. This included patients with mental health issues, ${ }^{36} 37$ nutritional concerns,${ }^{38}$ asthma, ${ }^{9} 293940$ diabetes, ${ }^{41-43}$ obesity, ${ }^{42}$ in hospital (ie, tertiary care),${ }^{44}$ with reproductive health concerns, ${ }^{45}$ who were being recruited for clinical trials ${ }^{2732}$ and for a study on spousal abuse. ${ }^{46}$ Study designs included prospective cohort studies, ${ }^{45}$ thematic analysis, ${ }^{37}$ qualitative surveys, interviews and focus groups, ${ }^{9} 27293239404346$ literature reviews, ${ }^{32} 38$ quantitative surveys, ${ }^{38} 42$ retrospective descriptive accounts of recruitment experiences, ${ }^{41}$ hermeneutic studies ${ }^{44}$ and mixed-methods syntheses. ${ }^{36}$

Most of the studies employed qualitative or survey-based techniques. As such, most outcome data are sourced from direct interview statements and personal experience. Limited empirical evidence on the effectiveness of the various recruitment strategies discussed was available. ${ }^{36} 384245$ Most of the included studies were conducted in the UK ${ }^{9} 2729236-43$ and dealt with recruitment of clinical populations to clinical trials. ${ }^{9} 29323639-43$

\section{Facilitators}

Three studies conducted in the $\mathrm{UK}^{27} 2942$ reported on facilitators of participation in research. Subjects perceived participation in research to be a possible route to improved treatment and health. ${ }^{27}{ }^{29}$ Participants also reported being motivated by the importance of disease prevention and potential to contribute to scientific 


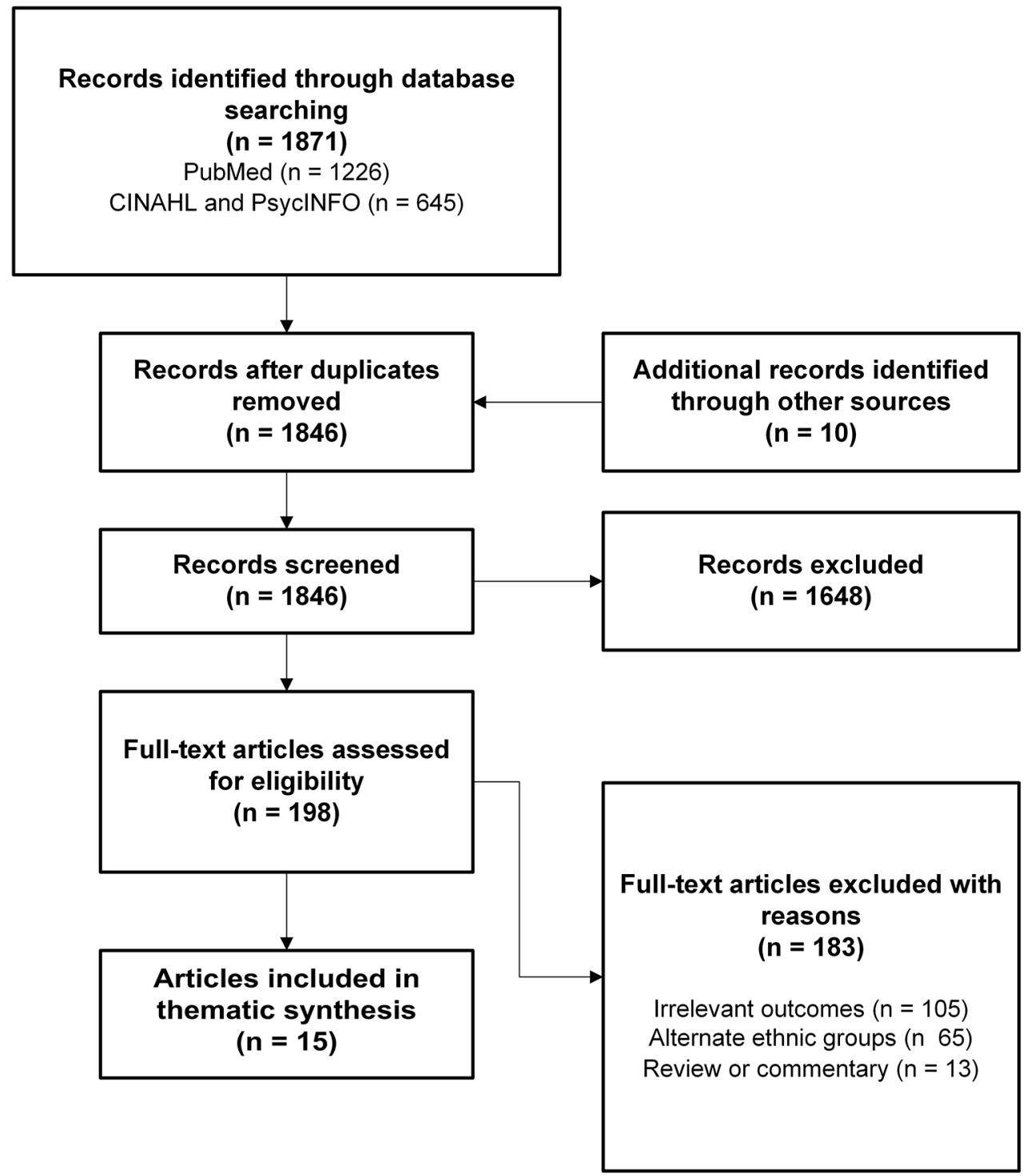

Figure 1 Flow chart of included and excluded studies.

knowledge, ${ }^{2742}$ by the potential to help society through participation ${ }^{27}$ and by a sense of obligation to healthcare providers. ${ }^{27}$ It was reported that higher social class and education, and younger age influence the level of awareness of clinical trials in South Asians. ${ }^{27}$

\section{Barriers}

Potential barriers to recruitment of South Asians are outlined in table 3. Participant-related factors followed themes of disinterest or lacking a feeling of belonging, conflicts, education or training-related deficits, logistical issues or opportunity cost, and factors related to fear or inhibition. Factors attributed to the researcher or research process followed themes of culture or language-related issues, logistical issues, issues related to study design and lack of awareness.

Treatment or participation-related factors included perception of risk of adverse effects (eg, treatment-related side effects), ${ }^{27} 293942$ fear of finding out health status $^{42}$ or experiencing the stigma of being labelled with a health condition, ${ }^{36}{ }^{39}$ previous poor experiences, ${ }^{27}$ mistrust of research, ${ }^{27} 3642$ inability to participate due to substance abuse or mental health issues ${ }^{36}$ and fear of being reported to immigration. ${ }^{36}$ Logistical concerns focused on cost of participating, ${ }^{3641}$ time away from work, family and other commitments, ${ }^{9} 27323637394142$ transportation and location, ${ }^{36}$ under-representation of South Asians at recruitment sites ${ }^{36}$ and lack of access to disease-specific services. ${ }^{36}$ Language-specific or culture-specific barriers included religious or cultural conflicts, ${ }^{32} 3637$ decisional hierarchies within families, ${ }^{27} 36$ lack of understanding about the consent process, ${ }^{36}$ researcher stereotypes about difficulties engaging with the South Asian population, ${ }^{36}$ inability to provide staff with language and cultural competency training, inability to translate study materials $^{9} 32363739$ and traditional gender roles. ${ }^{36} 37$ Some barriers related to general disinterest or lack of awareness, ${ }^{9} 3742$ including lack of interest due to previous treatment and the perception that participation would not 
Table 2 Study characteristics.

\begin{tabular}{|c|c|c|c|c|c|}
\hline First author, year & Country & Research area & Ethnic populations & Sample size & Study design \\
\hline Waheed, $2016^{36}$ & UK & $\begin{array}{l}\text { Mental Health } \\
\text { (Depression) }\end{array}$ & South Asians & Five studies $(\mathrm{n}=292)$ & Mixed-methods study \\
\hline $\begin{array}{l}\text { Garduno-Diaz, } \\
2014^{38}\end{array}$ & UK & Diet and Nutrition & South Asians & $\begin{array}{l}\mathrm{n}=300 \text { adults, } \mathrm{n}=100 \\
\text { children }\end{array}$ & $\begin{array}{l}\text { Literature review and } \\
\text { dietary survey }\end{array}$ \\
\hline Mac Neill, $2013^{29}$ & UK & $\begin{array}{l}\text { Asthma, Clinical } \\
\text { Trials }\end{array}$ & $\begin{array}{l}\text { Multiple ethnic groups } \\
\text { (primarily Bangladeshi) }\end{array}$ & $\begin{array}{l}n=42 \text { parents } \\
\text { ( } n=20 \text { Bangladeshi, } \\
n=22 \text { other) }\end{array}$ & Qualitative interviews \\
\hline Douglas, $2011^{41}$ & UK & Diabetes & South Asians & $n=1319$ potential recruits & $\begin{array}{l}\text { Descriptive review } \\
\text { of recruitment } \\
\text { experiences }\end{array}$ \\
\hline Stirland, $2011^{40}$ & USA/UK & Asthma & South Asians & $\mathrm{n}=36$ researchers & Qualitative interviews \\
\hline Sheikh, $2009^{9}$ & USA/UK & Asthma & South Asians & $\begin{array}{l}n=36 \text { researchers ( } 19 \text { UK, } \\
17 \text { USA), } n=10 \text { community } \\
\text { members }\end{array}$ & Qualitative interviews \\
\hline Lloyd, $2008^{43}$ & UK & Diabetes & $\begin{array}{l}\text { South Asians (Sylheti } \\
\text { and Mirpuri people from } \\
\text { Bangladesh) }\end{array}$ & $\mathrm{n}=31$ participants & Qualitative interviews \\
\hline $\begin{array}{l}\text { Mohammadi, } \\
2008^{44}\end{array}$ & Australia & $\begin{array}{l}\text { Hospitalised } \\
\text { Patients }\end{array}$ & Islamic South Asians & $n=13$ participants enrolled & $\begin{array}{l}\text { Interpretive } \\
\text { hermeneutic study }\end{array}$ \\
\hline Shelton, $2004^{46}$ & USA & Spousal Abuse & $\begin{array}{l}\text { South Asian women } \\
\text { (Bangladeshi) }\end{array}$ & $\begin{array}{l}\mathrm{n}=2 \text { researchers; number of } \\
\text { participants NR }\end{array}$ & Qualitative survey \\
\hline
\end{tabular}

*Some common data between publications.

NR, not reported.

confer any further benefit, ${ }^{3642}$ misgivings about the scientific importance of the work, ${ }^{9} 3742$ poor understanding of research intentions, ${ }^{9}$ perception of not belonging to the society standing to benefit from research, ${ }^{27}{ }^{32}$ simply not being approached to participate ${ }^{27}$ and researcher apathy towards achieving proper representation of South Asians. ${ }^{96}$ Finally, some study-specific issues included a lack of appropriate assessment tools (eg, translated or adapted tools) for South Asian populations, ${ }^{36} 37$ as well as narrow entry criteria (eg, restrictions on age and waist circumference). ${ }^{42}$

\section{Recruitment strategies}

Themes that emerged for recruitment strategies included language and culture-driven methods, communication and engagement strategies, logistical changes and accommodations, policy and study design measures, and compensation and incentives. The most commonly reported strategy was involvement of the South Asian community through mobilisation of key community figures or community partnerships. ${ }^{9} 273638414245$ The second most commonly reported strategies were incentives and reciprocal benefits ${ }^{96-40} 46$ and demonstrated respect and knowledge of South Asian culture, traditions and ethics. ${ }^{36-40} 4446$ Multiple studies also mentioned the development of trust and personal relationships, ${ }^{36} 3739404246$ the use of visual aids and reduced reliance on verbal exchange, ${ }^{29} 3843$ providing language support and translated materials, ${ }^{36} 384042$ personal 


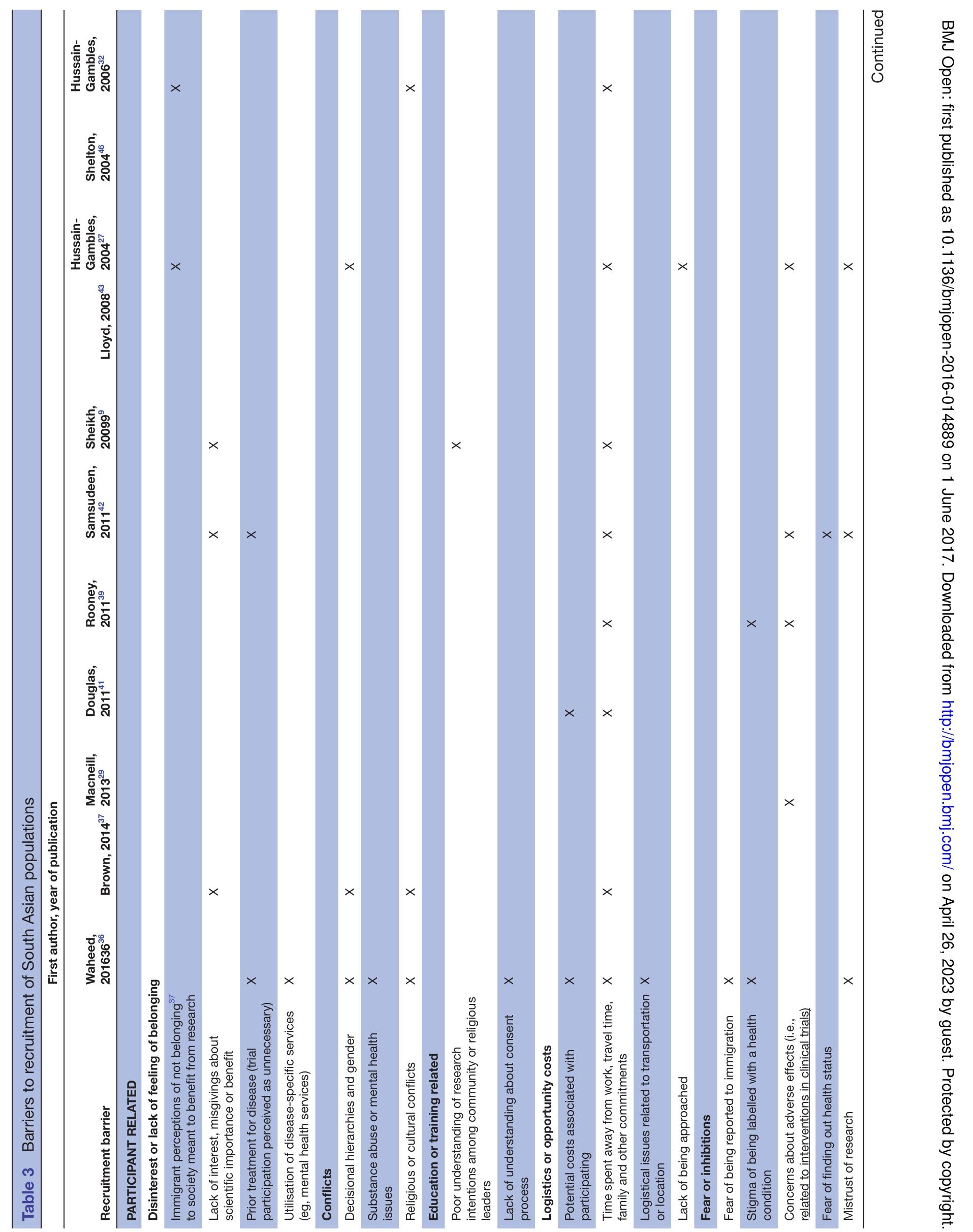




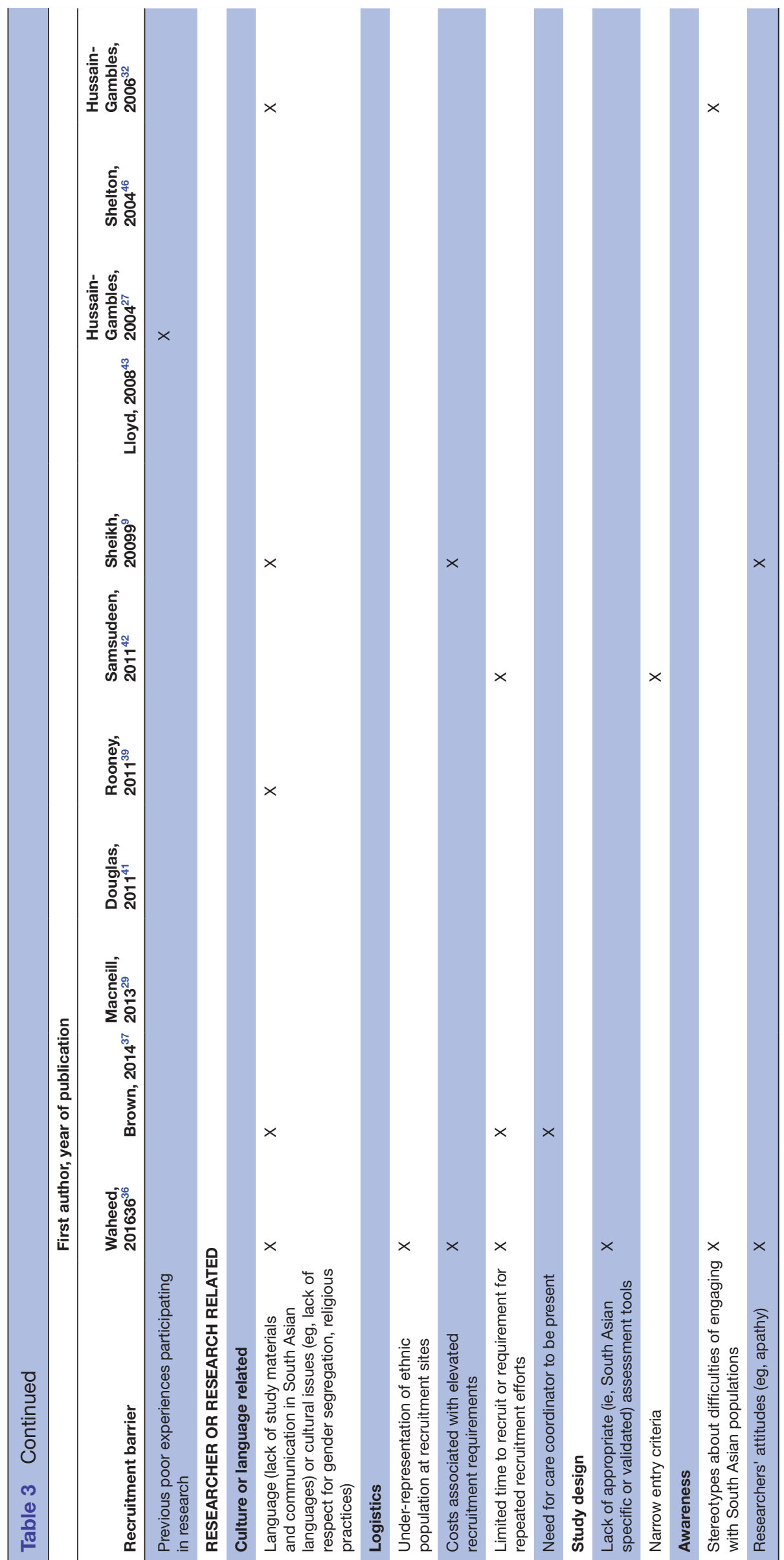

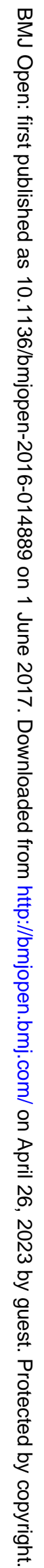


versus written contact, ${ }^{39} 4243$ training for staff in cultural competency, ${ }^{92736}$ conducting recruitment at places with high concentrations of South Asian attendance or residence $^{273638}$ and improving flexibility of appointment scheduling, location, childcare and transportation. ${ }^{36} 3940$ Several additional strategies for recruiting South Asian populations reported by two or fewer studies, as well as those previously discussed are outlined in table 4 .

\section{DISCUSSION}

To our knowledge, this is the first summary of evidence regarding factors that influence the participation of South Asians in health research studies. The studies summarised in this review identified multiple strategies to improve the success of recruitment efforts among South Asian populations. This information may help researchers to develop evidence-based strategies to improve representation of this minority group in health research.

Factors that facilitated South Asian participation in research included wanting to improve one's health and engage in disease prevention, to contribute to scientific knowledge and greater societal advances and a sense of obligation to healthcare providers. Interest in health and research may vary according to social class and education. ${ }^{27}$ Strategies that aim to capitalise on altruism and awareness $^{27} 32$ may systematically exclude individuals of lower socioeconomic status, resulting in recruitment bias, if there is a lack of perceived benefit to self. Benefits to self, including financial incentives, were factors associated with motivation to participate in research in Chinese patients in rural areas of lower socioeconomic status. ${ }^{47}$ Strategies such as using assessment tools with less emphasis on literacy, reducing participation costs and inconvenience and snowball sampling within broad South Asian communities could potentially mitigate this to some extent. ${ }^{48}$ Obligation to healthcare providers may be more common in South Asians than other ethnic groups. A literature review on South Asian perspectives on clinical and research ethics reported that medical paternalism persists in South Asian society. ${ }^{49}$ Recruitment involving senior physicians or researchers through partnerships between hospitals and academic centres is preferred to recruitment by individuals with perceived lesser hierarchical status by South Asians living in the UK. ${ }^{50}$ While this dynamic may be beneficial for increasing numbers where physician recruitment is feasible, potential abuse of this power dynamic may be detrimental. It may be necessary to have systems in place to limit exploitation of this relationship in the interest of reducing selection and performance bias. For instance, junior research staff could be involved in participant engagement until the stage that it is necessary for physicians to participate in the research process. Alternative methods of improving recruitment may be required where physician involvement is either not necessary or inappropriate.

Many of the barriers to participation in research relate to cultural insensitivity, lack of awareness of research or lack of efforts by the researchers to make contact, and tangible issues like time and cost of participating (table 3). Language was also a frequently cited issue. Language compatibility is reported to be of great importance to South Asian patients. ${ }^{51}$ Jolly et $a l^{20}$ observed a significantly higher proportion of South Asian individuals being excluded from participation in research due to language barriers compared with 'White Europeans' or those of 'Other' ethnicities. The use of multilingual research assistants, or principal investigators from the same ethnic background, or with language and cultural competency training is proposed as a possible strategy to overcome language barriers. This approach may also allow for larger recruitment pools if these staff are part of or able to access networks within the South Asian community. ${ }^{52}$ The effectiveness of this strategy was demonstrated in the successful recruitment and long-term retention of pregnant South Asian women for a study involving sample collection from mothers and their babies in the UK. ${ }^{53}$ The retention of study participants is another noted challenge for the South Asian population, ${ }^{27} 42$ not addressed by this scoping review.

Other common barriers are related to negative perceptions of researchers towards South Asian participants and vice versa. Various stereotypes held by health professionals conducting research on South Asian populations were highlighted as recruitment deterrents. ${ }^{932} 36$ Specific examples include perceptions or generalisations that South Asians are not punctual, that they have language limitations and that certain subpopulations (eg, seniors) would not have an interest in participating. ${ }^{932}$ In addition, perceived issues with gender hierarchies (eg, inability of women to make independent decisions), ${ }^{273637}$ the misconception that South Asians are less motivated about disease prevention $^{2454}$ and the association of English speaking with intelligence and potentially greater trial compatibility have been noted. ${ }^{32}$ On the other hand, South Asians reported that mistrust, mistreatment and disrespectful behaviour, sub-par care for non-English speakers and previous poor experiences participating in research would deter willingness to participate. ${ }^{32}$ Cross-cultural education of individuals working in research to dispel incorrect racial and cultural stereotypes, as well as education of South Asian communities to dispel some misconceptions about health research should be encouraged. ${ }^{55}$

From a logistical perspective, studies focused on South Asians may require upfront budgeting for enhanced recruitment strategies to address the unique barriers discussed. In particular, funds for multiple research sites, transportation of patients, incentives and childcare may need to be accounted for at the grant funding stages. Support for minority recruitment may be better in the USA where the National Institutes of Health Revitalization Act of 1993 promotes opportunities for women and minorities to participate in health research. ${ }^{567}$ There is mandated support for gender representation, but not for minority representation in Canada. ${ }^{58}$ In the UK and other European countries, the Research Governance 


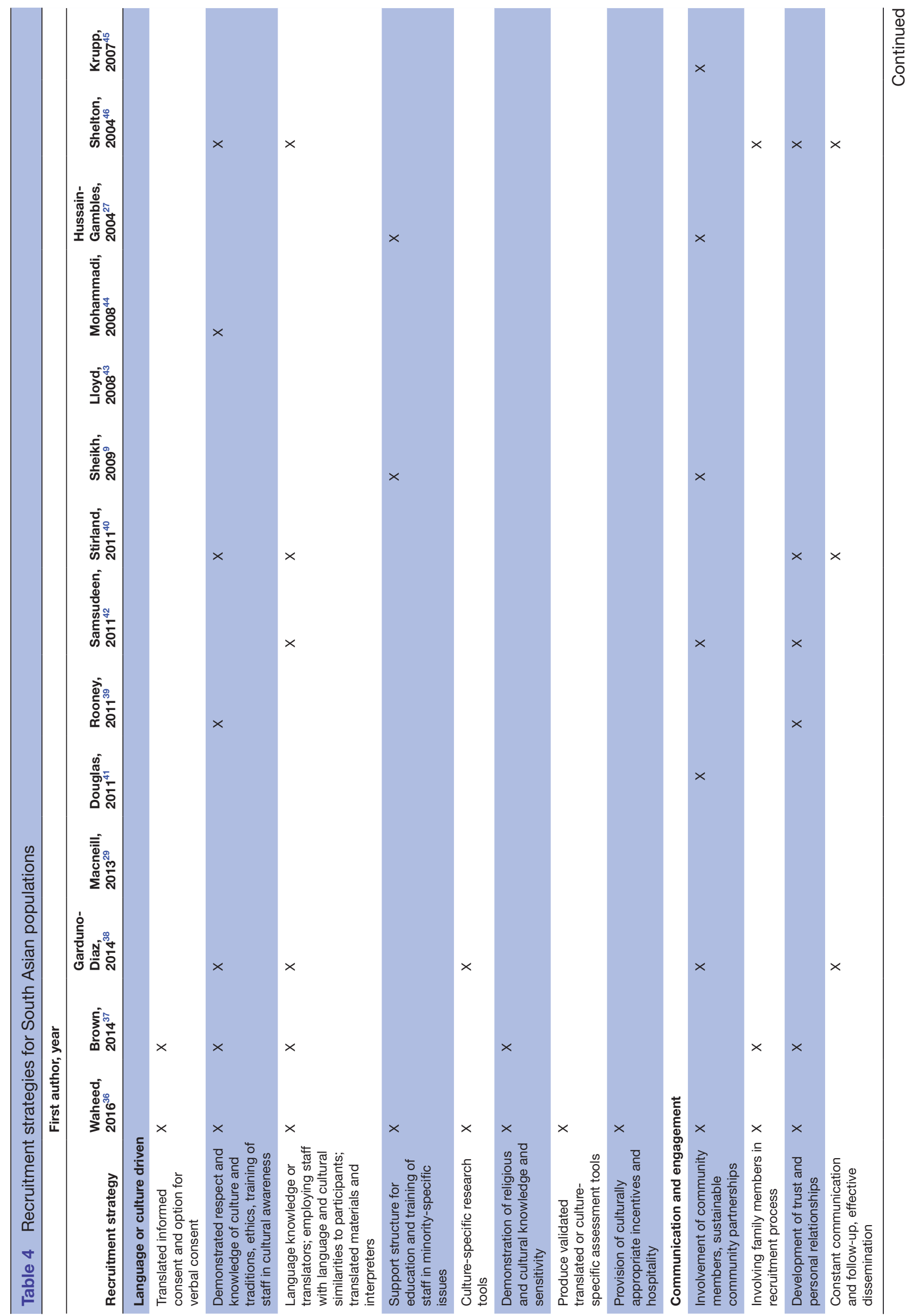

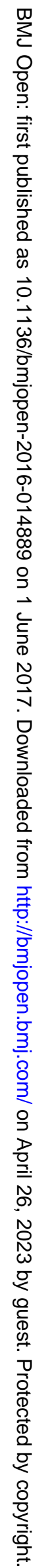




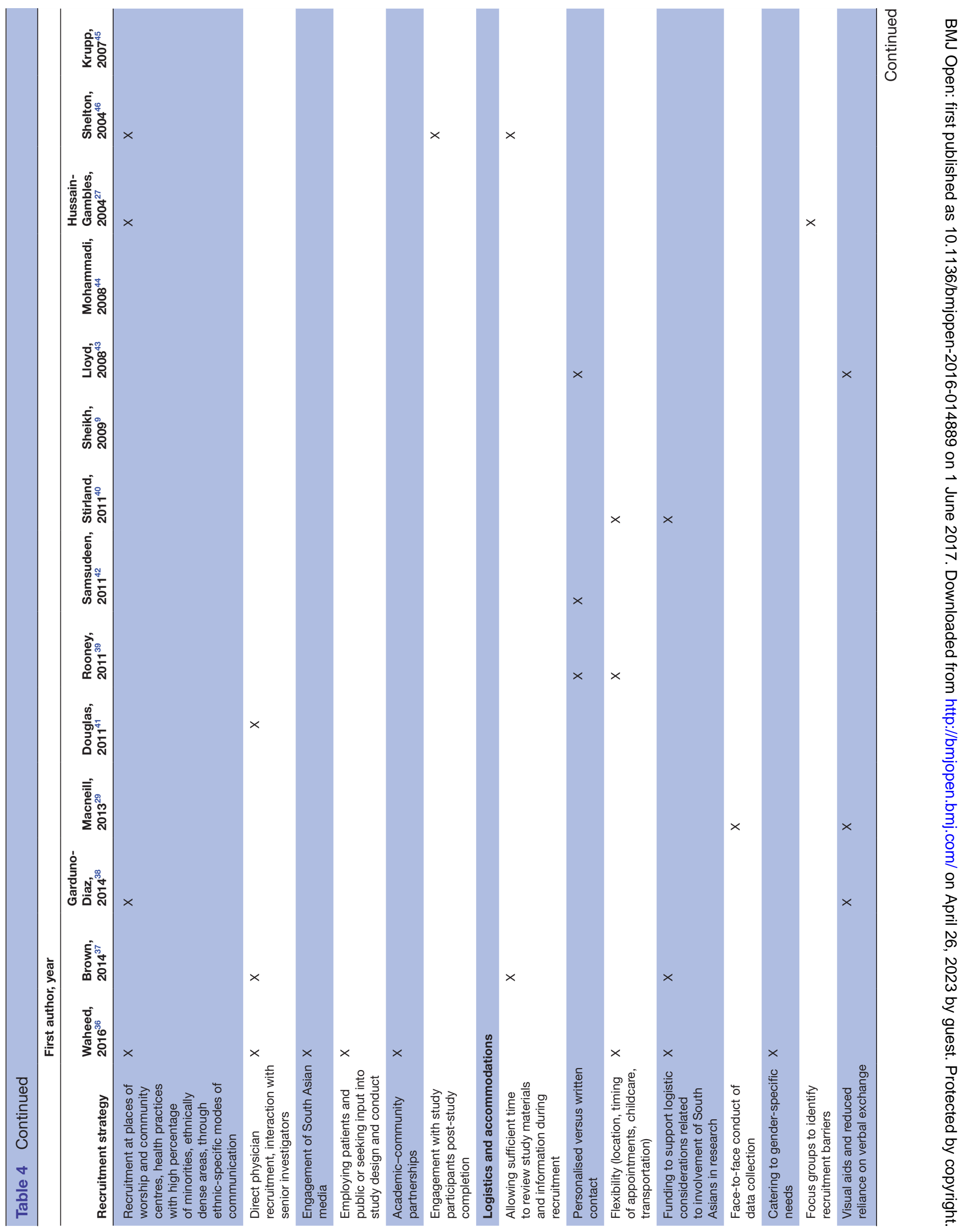




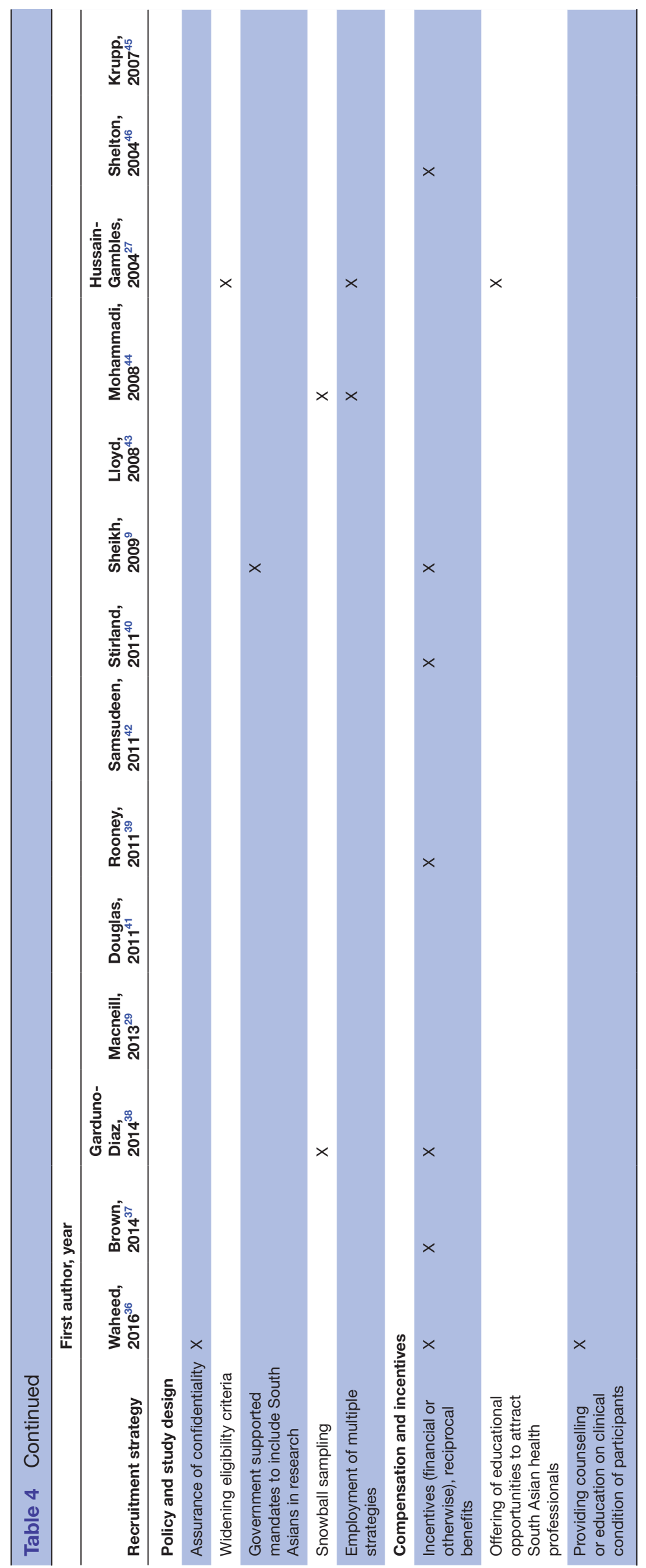

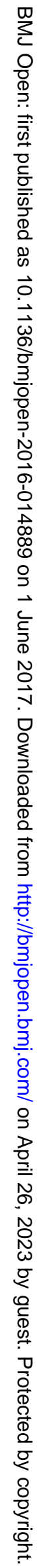


Framework encourages researchers to consider factors including race during research conduct when relevant, but it is not enforced. ${ }^{59} 60$ Government or institutional regulations promoting minority representation may encourage researchers to confront various barriers, despite the challenges involved.

There were similarities and differences in the findings of this scoping review in contrast to what is reported for other ethnic groups. Congruent with our findings, a systematic review of recruitment barriers and facilitators in African Americans, Latinos, Asian Americans and Pacific Islanders in the USA reported that issues of mistrust, competing demands, unintended outcomes (eg, adverse effects of intervention, lack of access to healthcare should injury or disease arise), lack of access to research information, stigma, health insurance coverage and jeopardising legal status in the USA were barriers to participation in research. ${ }^{26}$ Facilitators included cultural congruence, benefits to participation, altruism, convenience of participation and low risks associated with participation. They also found that there were issues specific to ethnic groups. For instance, the legacy of the Tuskegee Syphilis Study-an investigation noted for unethical abuse against African Americans-may discourage African Americans from participating in research, and Asian Americans often required the endorsement from family members. Another systematic review ${ }^{61}$ that assessed barriers and facilitators in indigenous populations in several countries, including the USA, Canada, Australia and New Zealand, reported that relationships and partnership, indigenous staff, indigenous knowledge models, targeted recruitment and adaptation of study material were associated with improved recruitment. Conversely, factors such as distrust of research attributable to the participants, study-centric issues such as trial design (no phone, travel costs) and lack of incorporation of indigenous knowledge systems dissuaded individuals from participating. While some barriers such as logistical issues may be common to multiple ethnic groups, the strategies to address cultural factors may differ depending on ethnicity. This highlights the importance of establishing targeted recruitment strategies that are not only specific to an individual ethnic group, but also consider heterogeneity between ethnic subgroups. This is pertinent to South Asians who as a group are multicultural, multilingual and multiethnic. ${ }^{62}$

A strength of this review is that a range of perspectives and ideas regarding recruitment of South Asians have been summarised and grouped thematically. Accordingly, this review represents a comprehensive in-depth overview of this subject area and provides a good estimate of the volume and characteristics of the published literature. Further, this review highlights areas for future investigation such as evaluation of the effectiveness of the proposed recruitment strategies in settings with large South Asian populations. There were several limitations to this review and the individual studies that were included. Published evidence was only available from the UK, the USA, India and Australia; therefore, transferability to the Canadian context may be limited. Also, the variable patient populations and sample sizes suggest that results may not be generalisable to all South Asian populations. In addition, there was a relative paucity of evidence regarding recruitment strategies for South Asians in these studies, and the majority of the evidence is in the context of clinical trials. The usefulness of the proposed recruitment strategies outside of the clinical trial setting is unknown. The applicability of the strategies presented may vary, as some proposed solutions are relatively straightforward, while others are more complex and difficult to apply. As discussed by Waheed $e t a l,{ }^{36}$ changing recruitment venues may be easy, but provision of appropriate incentives and catering to specific health and cultural beliefs may be more difficult to execute. The primary types of studies that have been used to investigate potential recruitment strategies in South Asian populations include focus groups and interviews. These studies provide valuable insight into participant and researcher perspectives, but do not directly address the quantitative impact of barriers or facilitators or effectiveness of recruitment strategies. Where empirical data exist, it is often limited to descriptive rates of recruitment and retention based on the results of quantitative surveys or prospective cohort studies. Documentation of barriers to recruitment in future studies including South Asian participants via qualitative interviews during the recruitment process would help to address this gap. As well, assessment tools, such as the Barriers to Research Participation Questionnaire, may assist in determining hurdles to research participation in specific populations in a structured manner. ${ }^{63}$ Since this was a scoping review aimed at mapping the current literature on this topic, we did not complete a quality assessment of the data. The search date restrictions that were imposed may have excluded studies with valuable historical perspectives. However, we believe this review captures a relevant snapshot of the most up-to-date research on this topic.

The evidence reviewed suggests that recruitment methods aimed at engaging with the target population may have a positive impact. One such intervention that was not discussed explicitly by the included studies is community-based participatory research. This research method aims to increase the mutual value of the research initiative for the researchers and the community. ${ }^{64}$ This approach employs strategies such as involving trusted community members as study staff, which may effectively mitigate ethical and cultural challenges in research. ${ }^{65}$ This approach may have the potential to mobilise individuals to take ownership of their health and encourage education and participation within their communities and to ensure appropriate dissemination of findings. ${ }^{66} 67$ One study in South Asian women concluded that participatory action research provided a platform for the participants to 'create and share knowledge' ${ }^{66}$ The overall paucity of evidence, particularly in the Canadian context, suggests that work is still needed to determine context-specific barriers and facilitators to recruitment and associated 
strategies to increase the participation of South Asians in health research. A mixed-methods synthesis from the Canadian perspective linking experiences of individuals and groups to the evidence on effectiveness of recruitment strategies may shed some light on potential approaches researchers could employ in future studies. Given the lack of empirical data, proposed recruitment strategies should be quantitatively evaluated, ideally using prospective experimental methods, to determine their relative effectiveness and value. Further, adequate representation of South Asians in research also relies heavily on the retention of participants, which has been reported to be variable in South Asians. ${ }^{68} 69$ The identification and investigation of retention strategies that ensure the successful recruitment is not undone should be of highest priority.

\section{CONCLUSIONS}

Better representation of South Asians in health research may promote development of tailored treatment and increased awareness and ownership of health. This would support the ultimate goals of improving the health of the South Asian population, reducing healthcare spending and addressing health inequity. The information presented in this review can be used to assist researchers when preparing to conduct research and may help to inform a more in-depth analysis of this issue from a Canadian perspective. Consideration of these issues during the grant writing and protocol stages of research may decrease the risk of encountering recruitment problems at latter stages.

Correction notice This paper has been amended since it was published Online First. Owing to a scripting error, some of the publisher names in the references were replaced with 'BMJ Publishing Group'. This only affected the full text version, not the PDF. We have since corrected these errors and the correct publishers have been inserted into the references.

Acknowledgements YL acknowledges funding from the Canada Research Chairs Program of the Canadian Institutes of Health Research. PAJ is supported by a Senior Scientist Salary Award from the British Columbia Children's Hospital Research Institute.

Contributors TAWQ, PAJ and YL were all involved in the conception of the review. TAWQ wrote the review protocol and TAWQ and LF were involved in conduct of the review. TAWQ wrote the initial draft. LF, PAJ, and YL were involved in reviewing the manuscript and in critical revision of the manuscript. All authors read and approved the final manuscript.

Competing interests TAWQ is an employee of the Canadian Agency for Drugs and Technologies in Health.

Provenance and peer review Not commissioned; externally peer reviewed.

Data sharing statement A copy of the unpublished study protocol is available upon request from the corresponding author.

Open Access This is an Open Access article distributed in accordance with the Creative Commons Attribution Non Commercial (CC BY-NC 4.0) license, which permits others to distribute, remix, adapt, build upon this work non-commercially, and license their derivative works on different terms, provided the original work is properly cited and the use is non-commercial. See: http://creativecommons.org/ licenses/by-nc/4.0/

(c) Article author(s) (or their employer(s) unless otherwise stated in the text of the article) 2017. All rights reserved. No commercial use is permitted unless otherwise expressly granted.

\section{REFERENCES}

1. Government of Canada SC. National household survey: immigration and ethnocultural diversity, 2014. http://www5.statcan.gc.ca/olc-cel/ olc. .action?Objld=99-010-X\&ObjType=2\&lang=en\&limit=1 (accessed 27 May 2015).

2. Ethnicity and national identity in England and Wales - Office for national statistics. $2012 \mathrm{http}: / /$ www.ons.gov.uk/peoplepopul ationandcommunity/culturalidentity/ethnicity/articles/ethnicitya ndnationalidentityinenglandandwales/2012-12-11 (accessed 19 Jun 2016).

3. Government of Canada SC. Classification of visible minority. 2009 http://www.statcan.gc.ca/eng/concepts/definitions/minority01a (accessed 17 Mar 2017).

4. Statistics Canada. Projections of the diversity of the Canadian population, 2013. http://www12.statcan.gc.ca/census-recensement/ 2011/ref/92-135/surveys-enquetes/vismin-population-minvis-eng. cfm (accessed 10 Jul 2014).

5. Veenstra G, Patterson AC. South Asian-White health inequalities in Canada: intersections with gender and immigrant status. Ethn Health 2016;21:639-48.

6. Gupta M, Singh N, Verma S. South Asians and cardiovascular risk: what clinicians should know. Circulation 2006;113:e924-e929.

7. Anand SS, Yusuf S, Vuksan V, et al. Differences in risk factors, atherosclerosis, and cardiovascular disease between ethnic groups in Canada: the study of Health Assessment and risk in ethnic groups (SHARE). Lancet 2000;356:279-84.

8. Rana A, de Souza RJ, Kandasamy S, et al. Cardiovascular risk among South Asians living in Canada: a systematic review and metaanalysis. CMAJ Open 2014;2:E183-E191.

9. Sheikh A, Halani L, Bhopal R, et al. Facilitating the recruitment of minority ethnic people into research: Qualitative Case Study of South Asians and asthma. PLoS Med 2009;6:e1000148.

10. Razak F, Anand SS, Shannon $\mathrm{H}$, et al. Defining obesity cut points in a multiethnic population. Circulation 2007;115:2111-8.

11. Twells LK, Gregory DM, Reddigan J, et al. Current and predicted prevalence of obesity in Canada: a trend analysis. CMAJ Open 2014;2:E18-E26.

12. Chiu M, Austin PC, Manuel DG, et al. Deriving ethnic-specific BMI cutoff points for assessing diabetes risk. Diabetes Care 2011;34:1741-8.

13. Nanditha A, Ma RC, Ramachandran A, et al. Diabetes in Asia and the Pacific: implications for the global epidemic. Diabetes Care 2016;39:472-85.

14. Sword W, Watt S, Krueger P. Postpartum health, service needs, and access to care experiences of immigrant and Canadian-born women. J Obstet Gynecol Neonatal Nurs 2006;35:717-27.

15. Nilaweera I, Doran F, Fisher J. Prevalence, nature and determinants of postpartum mental health problems among women who have migrated from South Asian to high-income countries: a systematic review of the evidence. J Affect Disord 2014;166:213-26.

16. Asanin J, Wilson K. "I spent nine years looking for a doctor": exploring access to health care among immigrants in Mississauga, Ontario, Canada. Soc Sci Med 2008;66:1271-83.

17. Hussain-Gambles M, Atkin K, Leese B. Why ethnic minority groups are under-represented in clinical trials: a review of the literature. Health Soc Care Community 2004;12:382-8.

18. Hilton BA, Grewal S, Popatia N, et al. The desi ways: traditional health practices of south asian women in Canada. Health Care Women Int 2001;22:553-67.

19. Bhandari N, Taneja S, Rongsen T, et al. Implementation of the WHO Multicentre Growth Reference Study in India. Food Nutr Bull 2004;25:S66-71.

20. Jolly K, Lip GY, Taylor RS, et al. Recruitment of ethnic minority patients to a cardiac rehabilitation trial: the Birmingham Rehabilitation Uptake Maximisation (BRUM) study [ISRCTN72884263]. BMC Med Res Methodol 2005;5:18.

21. Bajaj S, Jawad F, Islam N, et al. South Asian women with diabetes: psychosocial challenges and management: consensus statement. Indian J Endocrinol Metab 2013;17:548-62.

22. Wiseman N. Designations of medicines. Evid Based Complement Alternat Med 2004;1:327-9.

23. Statistics Canada. The South Asian community in Canada. 2007. http://www.statcan.gc.ca/pub/89-621-x/89-621-x2007006-eng.htm (accessed 19 Aug 2013).

24. Lucas A, Murray E, Kinra S. Heath beliefs of UK South Asians related to lifestyle diseases: a review of qualitative literature. $J$ Obes 2013;2013:1-13.

25. Grewal K, Stewart DE, Grace SL. Differences in social support and illness perceptions among South Asian and Caucasian patients with coronary artery disease. Heart Lung 2010;39:180-7. 
26. George S, Duran N, Norris K. A systematic review of barriers and facilitators to minority research participation among African Americans, Latinos, Asian Americans, and Pacific Islanders. Am J Public Health 2014;104:e16-e31.

27. Hussain-Gambles M, Leese B, Atkin K, et al. Involving South Asian patients in clinical trials. Health Technol Assess 2004;8:1-109.

28. Khan M, Kobayashi K, Lee SM, et al. (In)Visible minorities in Canadian health data and research. Population Change and Lifecourse Strategic Knowledge Cluster Discussion Paper Series 2015;3:5.

29. Macneill V, Nwokoro C, Griffiths C, et al. Recruiting ethnic minority participants to a clinical trial: a qualitative study. BMJ Open 2013;3:e002750.

30. Mason S, Hussain-Gambles M, Leese B, et al. Representation of South Asian people in randomised clinical trials: analysis of trials' data. BMJ 2003;326:1244-5.

31. Khunti K, Bellary S, Karamat MA, et al. Representation of people of South Asian origin in cardiovascular outcome trials of glucoselowering therapies in type 2 diabetes. Diabet Med 2017;34.

32. Hussain-Gambles M, Atkin K, Leese B. South Asian participation in clinical trials: the views of lay people and health professionals. Health Policy 2006;77:149-65.

33. Arksey H, O'Malley L. Scoping studies: towards a methodological framework. Int J Soc Res Methodol 2005;8:19-32.

34. Levac D, Colquhoun H, O'Brien KK. Scoping studies: advancing the methodology. Implement Sci 2010;5:69.

35. Balk EM, Chung M, Chen ML, et al. Assessing the accuracy of google translate to allow data extraction from trials published in Non-English languages. Rockville, MD: Agency for Healthcare Research and Quality, 2013. (accessed 17 Mar2017).

36. Waheed W, Husain N, Allen G, et al. Recruitment strategies for British South Asians in 5 depression trials: a mixed method study. J Affect Disord 2015;185:195-203.

37. Brown GE, Woodham A, Marshall M, et al. Recruiting South Asians into a UK Mental Health Randomised Controlled Trial: experiences of field researchers. J Racial Ethn Health Disparities 2014;1:181-93.

38. Garduño-Diaz SD, Husain W, Ashkanani F, et al. Meeting challenges related to the dietary assessment of ethnic minority populations. $J$ Hum Nutr Diet 2014;27:358-66.

39. Rooney LK, Bhopal R, Halani L, et al. Promoting recruitment of minority ethnic groups into research: qualitative study exploring the views of South Asian people with asthma. J Public Health 2011;33:604-15.

40. Stirland L, Halani L, Raj B, et al. Recruitment of South Asians into asthma research: qualitative study of UK and US researchers. Prim Care Respir J 2011;20:282-90.

41. Douglas A, Bhopal RS, Bhopal R, et al. Recruiting South Asians to a lifestyle intervention trial: experiences and lessons from PODOSA (Prevention of Diabetes \& Obesity in South Asians). Trials 2011:12:220.

42. Samsudeen BS, Douglas A, Bhopal RS. Challenges in recruiting South Asians into prevention trials: health professional and community recruiters' perceptions on the PODOSA trial. Public Health 2011;125:201-9.

43. Lloyd CE, Johnson MR, Mughal S, et al. Securing recruitment and obtaining informed consent in minority ethnic groups in the UK. BMC Health Serv Res 2008;8:68.

44. Mohammadi N, Jones T, Evans D. Participant recruitment from minority religious groups: the case of the Islamic population in South Australia. Int Nurs Rev 2008;55:393-8.

45. Krupp K, Madhivanan P, Karat C, et al. Novel recruitment strategies to increase participation of women in reproductive health research in India. Glob Public Health 2007;2:395-403.

46. Shelton AJ, Rianon NJ. Recruiting participants from a community of Bangladeshi immigrants for a study of spousal abuse: an appropriate cultural approach. Qual Health Res 2004;14:369-80.

47. Wu E, Wang T, Lin T, et al. A comparative study of patients' attitudes toward clinical research in the United States and urban and rural China. Clin Trans/ Sci 2015;8:123-31.
48. Yancey AK, Ortega AN, Kumanyika SK. Effective recruitment and retention of minority research participants. Annu Rev Public Health 2006;27:1-28.

49. Pratt B, Van C, Cong Y, et al. Perspectives from South and East Asia on clinical and research ethics: a literature review. J Empir Res Hum Res Ethics 2014;9:52-67.

50. Symonds RP, Lord K, Mitchell AJ, et al. Recruitment of ethnic minorities into Cancer clinical trials: experience from the front lines. Br J Cancer 2012;107:1017-21.

51. Ahmad F, Gupta H, Rawlins J, et al. Preferences for gender of family physician among Canadian European-descent and South-Asian immigrant women. Fam Pract 2002;19:146-53.

52. Lee SK, Sulaiman-Hill CR, Thompson SC. Overcoming language barriers in community-based research with refugee and migrant populations: options for using bilingual workers. BMC Int Health Hum Rights 2014;14:11.

53. Neelotpol S, Hay AW, Jolly AJ, et al. Challenges in collecting clinical samples for research from pregnant women of South Asian origin: evidence from a UK study. BMJ Open 2016;6:e010554.

54. Patel M, Phillips-Caesar E, Boutin-Foster C. Barriers to lifestyle behavioral change in migrant south asian populations. J Immigr Minor Health 2012;14:774-85.

55. Marshall A, Batten S. Researching across cultures: issues of ethics and power. Forum Qual Sozialforschung Forum Qual Soc Res 2004;5. http://www.qualitative-research.net/index.php/fqs/article/view/572

56. Epstein S. The politics of difference in medical research, 2001. http:// press.uchicago.edu/ucp/books/book/chicago///bo5414954.html (accessed 20 Jun 2016).

57. NIH guidelines on the inclusion of women and minorities as subjects in clinical research, 2001. https://grants.nih.gov/grants/funding/ women_min/guidelines_amended_10_2001.htm (accessed 20 Jun2016).

58. Canadian Women's Health Network. Report on governmental health research policies promoting gender or sex diferences sensitivity. Edmonton, AB: Institute of Gender and Health, 2003. http://www. cwhn.ca/en/node/25386 (accessed 20 Jun2016).

59. GOV.UK. Research governance framework for health and social care. 2nd edition. England: Department of Health. https://www.gov. uk/government/publications/research-governance-framework-forhealth-and-social-care-second-edition (accessed 31 Jan 2017).

60. Redwood S, Gill PS. Under-representation of minority ethnic groups in research--call for action. Br J Gen Pract 2013;63:342-3.

61. Glover M, Kira A, Johnston V, et al. A systematic review of barriers and facilitators to participation in randomized controlled trials by indigenous people from New Zealand, Australia, Canada and the United States. Glob Health Promot 2015;22:21-31.

62. Kallivayalil RA, Chadda RK, Culture CRK. Culture, ethics and medicine in South Asia. Int J Pers Centered Med 2011;1:56-61.

63. Kibler JL, Brisco K. Evaluation of a brief questionnaire for assessing barriers to Research Participation. Ethn Dis 2006;16:547-50.

64. Viswanathan M, Ammerman A. Community-based participatory Research: Assessing the Evidence: summary. US: Agency for Healthcare Research and Quality, 2017.

65. Minkler M. Community-based research partnerships: challenges and opportunities. J Urban Health 2005;82:ii3-ii12.

66. Choudhry UK, Jandu S, Mahal J, et al. Health promotion and participatory action research with south asian women. $J$ Nurs Scholarsh 2002;34:75-81.

67. Getrich CM, Sussman AL, Campbell-Voytal K, et al. Cultivating a cycle of trust with diverse communities in practice-based research: a report from PRIME net. Ann Fam Med 2013;11:550-8.

68. Kandula NR, Dave S, De Chavez PJ, et al. Translating a heart disease lifestyle intervention into the community: the South Asian Heart Lifestyle intervention (SAHELI) study; a randomized control trial. BMC Public Health 2015;15:1064.

69. Vlaar EM, van Valkengoed IG, Nierkens V, et al. Feasibility and effectiveness of a targeted diabetes prevention program for 18 to 60 -year-old South Asian migrants: design and methods of the DH!AAN study. BMC Public Health 2012;12:371. 